\title{
Using PRISM to profile semantic ability in Greek
}

Fani Nasika ${ }^{1}$, Katerina Afantenou ${ }^{2}$, Georgia Kremmyda ${ }^{3}$

${ }^{1}$ School of Allied Health Professions, Canterbury Christ Church University, UK

${ }^{2}$ School of Philosophy, Aristotle University of Thessaloniki, Greece

${ }^{3}$ School of Health, Aegean College, Greece

https://doi.org/10.36505/ExLing-2017/08/0020/000322

\begin{abstract}
This paper aims to show how PRISM-L and PRISM-G (Crystal, 1992) can be used to profile semantic ability or difficulties in Greek speakers. The two profiles were used for the analysis of three samples provided by one adult participant with aphasia and two normally developing children of 4;8 and 6;8 years old, respectively. The profiling procedure showed that PRISM can provided specific information regarding the range of the lexicon of an individual as well as details about the semantic analysis of their sentences. Based on PRISM, one can see the developmental course of a child and identify areas of clinical interest for further development or locate specific semantic difficulties at word and sentence level in adults with language impairments.
\end{abstract}

Key words: PRISM, semantic analysis, development, profile, Greek

\section{Theoretical Background}

Profiles of linguistic ability or disability are a useful tool for clinicians since they provide a means for identifying areas of weakness and/or strength regarding language in populations with developmental or acquired language disorders. Profiles are very important since the clinician can use them for assessing the language abilities of an individual, make diagnostic hypotheses and plan focused and structured interventions based on specific linguistic deficits identified in these profiles. Crystal (1992) created profile charts which could describe linguistic abilities in distinct linguistic levels of analysis, such as phonology (PROPH), prosody (PROP), morphosyntax (Language Assessment, Remediation and Screening Procedure, LARSP) and semantics (PRISM). In 2016, several cross-linguistic adaptations of LARSP were published, including a Greek version, the Gr-LARSP, by Stavrakaki and Okalidou (2016). Within this context, this study aims to adapt PRISM (Crystal, 1992) in order to profile semantic abilities and difficulties in Greek-speaking individuals.

PRISM comprises two separate procedures. The first concerns the relationship between semantics and the mental lexicon (PRISM-L) and the second the relationship between semantics and grammar (PRISM-G), mainly referring to thematic-role structures. PRISM-L includes a chart with an inventory of 61 semantic fields, divided into 239 semantic subfields, which is used to identify the lexical range of an individual as well as

ExLing 2017: Proceedings of 8th Tutorial and Research Workshop on Experimental Linguistics, 19-22 June, Heraklion, Crete, Greece 
the semantic areas he/she uses. Interestingly, these semantic fields are organized in an acquisition order. In addition, PRISM-L identifies relational items (e.g., pronouns, prepositions, connectives, etc.) as well as paradigmatic and syntagmatic relations and developmental errors.

On the other hand, PRISM-G is structured on the basis of five developmental stages, on sequences of semantic elements (e.g., Actor, Activity, etc.) and on semantic relationships (e.g., addition, contrast, condition, cause, etc.). It offers the clinician the possibility to identify thematic-role structures across various developmental stages and also their range and complexity. Finally, PRISM-G describes the order-of-mention of clauses and whether this parallels the order of the external world.

In the present study, PRISM-L and PRISM-G were used to profile the linguistic abilities and/or difficulties of Greek-speaking individuals.

\section{Methodology}

\section{Sample}

The data was collected from three participants, one adult and two children. The adult participant was 37 years old, male, and was diagnosed with Broca's aphasia, after a stroke. The two typically developing children participants included one girl ( $4 ; 8$ years old) and a boy (6;8 years old).

\section{Material and procedure}

The procedure involved the collection of language samples from the three participants. These samples involved the narration of the Red Riding Hood story. The adult participant provided a written language sample of the story while the two children provided oral language samples. The samples were analyzed using the PRISM-L and PRISM-G procedures, following Crystal (1992).

\section{Results}

Regarding the written sample of the adult participant, PRISM-L showed that he used 86 content words and 52 functional words. The type/token ratio (TTR) for the lexical items was 0.61 , which denotes a medium lexical range, while the TTR for the functional items was 0.41 , which shows a small range for functional words. Regarding the content words, the adult participant used words from 24 out of 61 semantic fields, namely time, buildings, living, man, measurement, moving, making/doing, animals, clothing, sound, sight, happening, body, etc. Of these semantic fields, the ones with the smallest lexical range were man, world and the animals. It can be seen that these categories are spread over the acquisition continuum which is understandable since people with aphasia may lose access to parts of an 
already acquired lexicon. Concerning PRISM-G, the adult participant produced mainly clauses of two or three semantic elements, which places him in Stages II and III. The most frequent combinations of basic functions and thematic roles that he produced were combinations of dynamic and stative verbs with temporal or locative semantic elements. Very often, he omitted the Actor of the action or the Experiencer or the thematic roles were reversed. Additionally, coordination and subordination were minimal.

On the other hand, the boy produced 53 function words, with a TTR of 0.3 , and 66 content words, with a TTR of 0.6. In addition, he produced words from 19 out of 61 semantic fields while not producing words from the last 12 semantic fields of PRISM-L, which correspond to later stages of lexical development. The semantic fields covered were mostly man, moving, food, having, animals, thinking, feeling, clothing, furniture, make/do, size, body, building. Within these semantic fields, he produced words from various sub-fields, showing an evolving and developing vocabulary. Regarding PRISM-G, the boy produced clauses with four, three and two semantic elements, showing also coordination and subordination of two or more clauses. The main semantic relations presented at the clause level were addition, time, cause, location and purpose. The functions and thematic roles of the semantic elements were unambiguous and covered a wide range of combinations. According to Crystal (1992), the boy's profile matches Stage IV of PRISM-G and presents many elements of Stage V.

Finally, the girl produced 94 function words, with a TTR of 0.2 , and 79 content words, with a TTR of 0.6 , indicating a lot of repetition and a limited range of function words along with a wider range of vocabulary in the content words. The words produced by the girl included 18 out of 61 semantic fields, consisting mostly of the following: man, moving, quantity, procession, animals, food, language, sight. The semantic fields covered did not include the final 12 fields of PRISM-L, which are considered to be acquired later. As far as PRISM-G is concerned, the girl produces clauses with three semantic elements, with various combinations of thematic roles and functions. Moreover, she uses coordination extensively with 'and', linking together two, three or more clauses. Regarding subordination, it is infrequent while she uses only relative pronouns, such as 'which'. Thus, the profile of the girl matches Stage III, as far as clausal semantic elements are concerned, and presents a developing coordination and subordination system from Stage V.

\section{Conclusions}

The data analysis showed two different profiles: the one presented by the adult participant and the ones presented by the two normally developing children. Regarding the two children, it can be seen that the older the child, 
the better they use function words and construct semantically complex sentences with subordination. As for the semantic fields acquired, it seems that the later fields of PRISM-L are not used extensively by young children and it may be the case that these lexical items are acquired and/or consolidated later. On the other hand, the adult profile showed elements of an already acquired lexical system, since he used words from various semantic fields, even from the ones found later in PRISM-L. However, the range of this vocabulary was medium which can be explained by the language impairment the adult has suffered due to his aphasia. In addition, his sentences contained mostly two or three semantic elements, which is lower than that of the $4 ; 8$ year-old girl, while he does not use complex sentence structures, either by coordination or subordination of clauses. This is also typical of individuals with aphasia and shows that PRISM-G can profile this difficulty.

To conclude, PRISM seems able to provide useful information on the development of semantic abilities in typically developing children and it can delineate the semantic profiles of individuals with aphasia, thus, providing an insight to their lexical range, semantic fields and their ability to form semantically sound sentences, which could inform clinical assessment and intervention.

\section{References}

Crystal, D. 1992. Profiling linguistic disability. London, Whurr Publishers.

Stavrakaki, S., Okalidou, A. 2016. Gr-LARSP: Towards a Greek version of LARSP. In Fletcher, P., Ball, M. J., Crystal, D. (eds.) 2016, Profiling Grammar: More Languages of LARSP. Multilingual Matters. 